\title{
Problems involved in elimination of both the singularity in the solution of nonlinear equation of motion on the rotation axis and the unbalance caused by convective heat transfer
}

\begin{abstract}
Yu. V. Vandakurov and E. M. Sklyarova
A. F. Ioffe Physicotechnical Institute, Russian Academy of Sciences, St. Petersburg, 194021

Russia, email: yv.mhd@mail.ioffe.ru

We are considering below some complex problems which arise in theoretical description of the processes developing in convective zones of rotating stars. It is common knowledge that the solutions of the starting nonlinear equations of equilibrium or motion may have singularities at the rotation axis, whose elimination in the case of equations of an even more general nature including spins was treated in quantum mechanics by invoking a numerical procedure (see, e.g., Varshalovich et al. (1988)). If spin effects are disregarded, these equations, which describe general representations of conventional and Alfven velocities as expansions in orthogonal vector spherical harmonics can be cast in an analytical form Vandakurov (1999).

The pattern of the self-establishing differential rotation of the convective zone should obviously be related to the heat transport conditions. It is known, for instance, that in order for radiative heat transport to set in in a rotating star, Eddington-Sweet circulation has to be excited (see discussion in Tassoul (1978)). Consideration of convective heat transport likewise meets with a specific difficulty associated with the fact that any radial displacement of a convective element in a rotating medium (other than parallel to the rotation axis) is intimately connected with generation of an unbalanced azimuthal force Vandakurov (2003)). Thus, the condition of azimuthal balance in a nonviscous rotating
\end{abstract} medium can be written in a nonmagnetic case as

$$
\frac{\partial v_{\varphi}}{\partial t}+[(\boldsymbol{\nabla} \times \mathbf{v}) \times \mathbf{v}] \cdot \mathbf{i}_{\varphi}=0
$$

where $\mathbf{v}$ is the velocity, $t$ is the time, $\varphi$ is the azimuthal angle, and $\mathbf{i}_{\varphi}$ is the azimuthal unit vector. For instance, in a solid body rotating with an angular velocity $\Omega$ medium, $\nabla \times \mathbf{v}=\mathbf{i}_{z} 2 \Omega$, where the $z$ axis is parallel to the rotation axis, so that in the case of a radially moving (with velocity $v_{r}$ ) convective element Eq. (0.1) should contain an azimuthal force. This force is nonzero everywhere except at the poles.

Such an azimuthal force is generated clearly in any differential rotation pattern of the medium. For instance, the radial velocity $v_{r}$ at the bottom of the solar convection zone was estimated Spruit (1974) to be of the order of $10^{3}-10^{4} \mathrm{~cm} / \mathrm{s}$. This yields a year to a month for the scale time of variation of the rotation velocity $v_{\varphi}$, if, as in the Sun, $v_{\varphi}$ is $2 \mathrm{~km} / \mathrm{s}$. In actual fact, this force is naturally generated in ascending heated or descending cooler flows of plasma; nevertheless, an unbalanced side force may probably bring about noticeable deviations of convective elements from their radial motion and, hence, initiation of chaotic variations in the rotation velocity, which would interfere with convective heat transport. Thus, processes which favor ordering of convective heat transport in stars may play an important role. 
We consider the solution to this problem under the assumption that additional forces may appear in the convective zone as a result of self-excitation of longitude-dependent toroidal fields (which contain both conventional and Alfven velocities). Calculations Vandakurov (2003) show that these longitude-dependent fields can be neutrally stable in a rotating medium in the presence of an axisymmetric toroidal magnetic field whose latitude dependence is governed by the $\sin (2 \theta)$ function. For example, for the bottom of the solar convection zone (with the density $\sim 0.1 \mathrm{~g} / \mathrm{cm}^{3}$ ) the maximum value of this field is about $110 \mathrm{kG}$.

\section{References}

Spruit, H.C. 1974, Solar Phys. 34, 277.

Tassoul, J.-L. 1978, Theory of Rotating Stars Princeton Univ. Princeton, New Jersey

Vandakurov, Yu.V. 1999, Astron. Rep. 43, 24

Vandakurov, Yu.V. 2003, Tech. Phys. 48, 298

Varshalovich, D.A., Moskalev, A.N., Khersonskii, V.K. 1988, Quantum theory of angular momentum, World Scientific Publishing Co.,Singapore. 